\title{
Network Pharmacology Approach and Experimental Verification of Huashi Dingtong Decoction Against Knee Osteoarthritis
}

\section{Ge Hai-Ya}

Fujian University of Traditional Chinese Medicine https://orcid.org/0000-0002-8230-4387

\section{Yan Lai-Jun}

Fujian University of Traditional Chinese Medicine

\section{Zhang Yan}

Fujian University of Traditional Chinese Medicine

\section{Zhang Ying-Sheng}

Fujian University of Traditional Chinese Medicine

\section{Lai Yu-Yang}

Fujian University of Traditional Chinese Medicine

\section{Geng Qiu-Dong}

Fujian University of Traditional Chinese Medicine

\section{Huang Ze-Ling}

Fujian University of Traditional Chinese Medicine

Li Nan ( $\triangle$ mr.linan@126.com )

Fujian University of Traditional Chinese Medicine

\section{Research}

Keywords: Knee osteoarthritis, inflammation, p38MAPK, Network pharmacology, Huashi Dingtong decocion

Posted Date: August 26th, 2020

DOl: https://doi.org/10.21203/rs.3.rs-62908/v1

License: (c) (i) This work is licensed under a Creative Commons Attribution 4.0 International License. Read Full License 


\section{Abstract}

Background: The aim of this study is to clarify the ingredients and targets of HSDTT against KOA by network pharmacology,and to verify the mechanism of HSDTT in treatment of KOA in vivo.

Methods: Ingredient-target network for HSDTT and KOA was created to identify the potential targets, protein-protein interaction network was used to find the key targets of HSDTT in treatment for KOA, GO enrichment and KEGG pathway was conducted to illuminate the pathway related to KOA treat by HSDTT. Rat model of KOA was established by joint injection in papain. The morphology of cartilage were assessed by H\&E. ELISA was used to detect the contents of inflammation cytokines in synovial fluid and synovium. The expression of the pathway protein were assessed by PCR.

Results: The results of network pharmacology demonstrate that there are 440 ingredients of HSDTT against knee osteoarthritis by 478 targets. The KEGG enrichment analysis showed that PI3K-Akt signaling pathway, MAPK signaling pathway were the key pathways for HSDTT to treat KOA. Morphology of cartilage was improved in the HSDTT group when compared with the model group. Our experiment show that HSDTT can reduced the expressions of p38 and p53 in cartilage, increased the expression of collagen $\bigotimes$. The contents of IL- $1 \beta$ and TNF- $\alpha$ in the synovium and COX-2 and PGE- 2 in synovial fluid were decreased significantly in the HSDTT group when compared with the model group.

Conclusions: Our study indicadites that HSDTT is capable to alleviate inflammation and delay the progression of KOA by p38MAPK signaling pathway.

\section{Introduction}

Knee osteoarthritis (KOA) is a chronic articular disease which characterized by cartilaginous degeneration, synovial membrane inflammation, hyperosteosis of subchondral bones.Most of KOA patients go to hospital for joint pain, stiffness, swelling, and dysfunction[1, 2]. Without timely and effective treatment, it will lead to different degrees of malformation in joint or even disability with the development of this disease[3]. It is reported in an epidemiological study that KOA is the main disease which acount for $7.1 \%$ among musculoskeletal disorders[4]. Furthermore, in a multivariate analysis, disability was positively related with cardiovascular events and all-cause mortality among patients with $\mathrm{KOA}[5]$. Therefore, how to treat KOA effectively and delay the progression of it is an urgent problem at the present stage.

In the past few decades, most of the experts have focused on chondropotection when they treated $\mathrm{KOA}[6]$. However, in recent years, many clinical researches show that KOA is not just a disease related to cartilage degradation but a whole jiont disease which involving cartilage, synovial membrane, ligaments, bones, muscles[7]. Furthermore, while KOA has been classified as non-inflammatory disease for a long time, the degree of inflammation is now considered as a critical factor in KOA pathology[8]. Several studies indicated that in the early-stage of KOA, many inflammation cytokines like tumor necrosis factor 
alpha (TNF-a) and interleukin-1 beta ( IL-1 $1 \beta$ ), which triggered by factors such as biomechanical stress , will active the signaling pathways and then in turn accelerate the progression of $\mathrm{KOA}[9]$.

Mitogenactivated protein kinase (MAPK) is one of the signaling pathways in cartilage which regulates the expression of proinflammatory cytokines[10]. The cascades of this signaling pathway include p38 MAPK, extracellular regulated kinase (ERK) and c-Jun N-terminal kinase (JNK)[11]. p38 MAPK is considered as a positive regulator in differentiation, inflammation and apoptosis of chondrocyte[12, 13]. Once the cartilage was damaged, the fragments of it will trigger the expression of the inflammatory cytokines, and further affect the synovial membrane and induce the degradation of cartilage by activiatng the protein kinases of this pathway[10].

Huashi Dingtong decoction(HSDTT) is a traditional Chinese medicine (TCM) formula, which consists of one protein and eight herbs: Squama Manis, Cinnanmomi Cortex, Rhizoma Dioscoreae, Angelicae Sinensis Radix ,Cortex Acanthopanacis, Radix Angelicae Biseratae ,Notopterygii Rhizoma Et Radix, Atractylodes lancea(Thumb.)DC ,Licorice. HSDTT was presented in the 'Orthopedics Experience' formulary written by Rugao Lin, a famous doctor of Traditional Chinese Osteopathy \& Traumatology and was used to treat KOA commonly by TCM doctors. However, the potential mechanism that HSDTT delay the progression of KOA remains unknown. In our study, we try to use network pharmacology method to present the drug-ingredient-target network of HSDTT and analyse the specific mechanism of the HSDTT effects on KOA. Finally, an animal experiment was conducted to verify whether HSDTT would alleviate inflammation and delay the development of KOA based on the result of the network pharmacology.

\section{Methods}

\subsection{Investigation of the mechanism of HSDTT against KOA using network pharmacology}

\subsubsection{Data preparation}

Huashi Dingtong decoction(HSDTT) was consist of Squama Manis (Chuan-Shan-Jia,CSJ) ,Cinnanmomi Cortex(Rou-Gui,RG), Rhizoma Dioscoreae (Shan-Yao,SY) , Angelicae Sinensis Radix (Dang-Gui,DG) ,Cortex Acanthopanacis (Wu-Jia-Pi,WJP), Radix Angelicae Biseratae (Du-Huo,DH) ,Notopterygii Rhizoma Et Radix(Qiang-Huo,QH) , Atractylodes lancea(Thumb.)DC (Cang-Zhu,CZ), licorice (Gan-Cao,GZ). Each ingredient of Chinese herbs were seached from other papers, Chemical database (http://www.organchem.csdb.cn)[14] and TCMSP database (https://tcmspw.com/index.php)[15]. All the 2D structure of ingredients were download from TCMSP database and pubchem(https://pubchem.ncbi.nIm.nih.gov/)[15,16]. The inclusion criterias of these ingredients are assessed by SwissADME (http://www.swissadme.ch/index.php)[17]. If the uploading ingredient in SwissADME meet the rules that it shows "high" in GI absorption of "Pharmacokinetics " and more than two "yes" in "Druglikeness", we will bring it into our study[18]. KOA-related genes were obtained from TTD database (https://db.idrblab.org/ttd)[19],OMIMdatabase(https://www.omim.org/) [20],Genecardsdatabase(https://www.genecards.org)[21]. 


\subsubsection{Drug-ingredients-targets network}

The targets, which "probability" $>0$, of included active ingredients were selected by Swiss TargetPrediction (http://www.swisstargetprediction.ch/)[22]. The drugs-ingredients-targets network was established by Cytoscape 8.0.0. The degree of each node in the network was analyzed by using Network Analyzer Tool in cytoscape[23].

\subsubsection{Protein-protein interaction (PPI) network construction and analysis}

The selected targets were uploaded in STRING databases(http://string-db.org) to confirm the potential proteins interactions[24]. Then the file named 'interaction' was put into Cytoscape 8.0.0 to construct the PPI network. The degree and combind-score analyzed by 'Network Analyzer Tool' indicated the importance of each target. Gene ontology (GO) enrichment and Kyoto Encyclopedia of Genes and Genomes (KEGG) pathway analysis were performed in $\mathrm{R}$ with the clusterProfiler package[25].

\subsection{Chemicals and reagents}

Huashi Dingtong decoction was purchased from TCM Pharmacy of the third hospital affiliated to Fujian University of Traditional Chinese Medicine. Ethyl carbamate and Hematoxylin-Eosin staining kits was obtained from Beijing Solarbio Science \& Technology Co., Ltd (Beijing,China). $500 \mathrm{U}$ of type II collagenase from clostridium histolyticum was purchased from Sigma-Aldrich. 0.9\% Sodium Chloride Injection was from Jiangxi Kelun Pharmaceutical Co.,Ltd (Jiangxi,China). ELISA kits of IL-1 $\beta, T N F-a, C O X-2, P G E-2$ was provided by Shanghai Westang Bio-tech co.,Ltd (Shanghai,China).

\subsection{Preparation of HSDTT}

HSDTT is consist of Squama Manis (Chuan-Shan-Jia,6g), Cinnanmomi Cortex(Rou-Gui,1g), Rhizoma Dioscoreae (Shan-Yao,9g), Angelicae Sinensis Radix (Dang-Gui,6g), Cortex Acanthopanacis (Wu-JiaPi,9g), Radix Angelicae Biseratae (Du-Huo,6g), Notopterygii Rhizoma Et Radix(Qiang-Huo,6g), Atractylodes lancea(Thumb.)DC(Cang-Zhu,6g), licorice(Gan-Cao,3g). The botanical name of these medicines has been checked with http://www.organchem.csdb.cn,respectivly.

\subsection{Animals experiments, grouping and model establishment}

32 male Sprague-Dawley (SD) rats weighing 180-220g were provided by Shanghai SLAC Laboratory Animal Co.,Ltd (Shanghai, China),the certificate number is SCXK 2017-0005. Rats housed in SPF facility were randomly divided into 3 groups: HSDTT group, Model group, Control group.There were 12 rats in Control group and Model Group, 8 rats in HSDTT group. Rats in HSDTT group were received HSDTT decoction at a dose of $4.68 \mathrm{~g} / \mathrm{kg} / \mathrm{d}$ by intragastric administration for 28 days after model successfully established. The other two groups were recived saline for the same days. The dose of intragastric administration was calculated by the surface area of the body. 
KOA model was established by intra-articular injection with type II collagenase $(0.4 \mathrm{mg} / \mathrm{ml})$. Rats in model group and HSDTT group were injected $(0.4 \mathrm{ml})$ into the medial side of the right knee joint of the hind limbs in the first, fourth day. The control group was injected with $0.4 \mathrm{~mL}$ saline. After the model estabilshed successfully for 1 week, four rats were randomly selected to identificate and evaluate the model in each group except HSDTT group. The other rats were sacrificed after intragastric administration for 28 days. The procedures of this reseach complied with the Chinese Animal Welfare Law and approved by Fujian University of Chineses Traditional Medicine (Protocol ID:NO. FUTCM-2019028).

\subsection{Measurement of the Knee Joint Diameter}

The transverse diameters of the right knees were measured at day0, day7,day14,day21,day28,day35 by digimatic caliper (Mitutoyo, Kanagawa, Japan).The knee joints were flexed at $90^{\circ}$ and measured the distance between the left and right highest points of the knee.

\subsection{Hematoxylin-eosin staining (H\&E)}

The right hind knee joint of rats were dissected and fixed in $10 \%$ formalin for $72 \mathrm{~h}$ at room temperature. Then tissues were decalcified in ethylenediaminetetraacetic acid for 6 weeks. Subsequently, knee joints were routinely sliced $(5 \mu \mathrm{m})$ after dehydration and paraffin imbedding. Finally, the slices were stained with hematoxylin-eosin staining kit and observed under a light microscope (magnification, 100x).

\subsection{Enzyme-linked immunosorbent assays (ELISA)}

The contents of IL-1 $\beta$ and TNF- $\alpha$ in the synovium and COX-2 and PGE-2 in synovial fluid were measured with ELISA kits. In brief, the procedure to test for IL-1 $\beta, T N F-a, C O X-2$ and PGE-2 was followed by the manufacturer's instructions. Each group was selected 3 rats to assay and all the samples were assessed for three times.

\subsection{Polymerase Chain Reaction (PCR)}

The cartilage tissues were used to isolate total RNA by RNAiso reagents. PrimeScript@RT Master Mix Perfect Real Time kit was performed to reverse transcription of total RNA. PCR amplification was carried out using gene specific.PCR primers were provided by Shanghai biosune co. (Shanghai, China),which

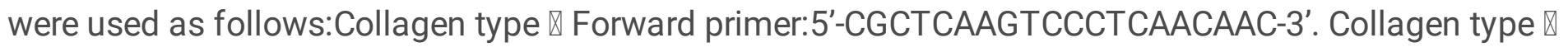
Reverse primer: 5'-TATCCAGTAGTCACCGCTCTTC-3'. p53 Forward primer: 5'-CAGCACATGACGGAGGTTGT3'. p53 Reverse primer:5'-TCATCCAAATACTCCACACGC-3'. p38 Forward primer: 5'TGTGATTGGTCTGTTGGATGTG-3'. p38 Reverse primer: 5'-TGGATTATGTCAGCCGAGTGTA-3'.

\subsection{Statistical analysis}

All the experimental data are expressed as the mean \pm SD. Two-sample $t$-test or Wilcoxon rank sum test were used to compare the difference between the two groups by software SPSS20.0. The significant difference was dicided by $p<0.05$. 


\section{Results}

\subsection{Investigation of the mechanism of HSDTT against KOA by network pharmacology}

\subsubsection{HSDTT ingredient-target network construction}

A total of 440 HSDTT-related ingredients were obtained from papers and Chemistry Database which include 8 Squama Manis (Chuan-Shan-Jia) components, 10 Cinnanmomi Cortex(Rou-Gui) components, 48 Rhizoma Dioscoreae (Shan-Yao) components, 74 Angelicae Sinensis Radix (Dang-Gui) components, 6 Cortex Acanthopanacis (Wu-Jia-Pi) components, 47 Radix Angelicae Biseratae (Du-Huo) componets, 81 Notopterygii Rhizoma Et Radix(Qiang-Huo) components, 21 Atractylodes lancea(Thumb.)DC components, 197 Licorice(Gan-Cao) components shown in table S1. Total 3096 KOA-associated genes were searched from TTD/GeneCards/OMIM databases. As is shown in Fig. 2 and Table S2, 478 genes which were ralated to HSDTT and KOA, were identified with Venn digram. Considering the large number of these genes, the first 200 genes were chosen to construct the drugs-ingredients-targets network with Cytoscape 8.0.0 software. It is composed of 605 nodes, which contain 9 chinese herbs, 403 ingredients, 200 targets. Hexagons represent different ingredients, circles represent different herbs, rhombus represent different targets(Fig.3).

\subsubsection{PPI network construction and core target screening}

The obtained potential therapeutic targets associated with KOA and HSDTT were input into String database.Then the file about PPI network from String database were input into Cytoscape 8.0.0 software. There were 478 nodes and 11506 edges in network. The larger and darker color nodes indicates the greater degree of targets. The thicker and the darker color lines represent the larger combined-score between targets(Fig. 4).

\subsubsection{Enrichment of GO analysis and KEGG pathway analysis}

To analyse the enrichment of Gene Ontology (GO) and KEGG pathway of 478 targets, the R with clusterprofiler package was used to make the results more visualized.As is shown in the Fig.5A, the top 3 functions of $\mathrm{GO}$ enrichment, which is related to protein binding and biological process, were protein tyrosine kinase activity,protein serine/threonine kinase activity,transmembrane receptor protein kinase activity.The KEGG enrichment, which is associated with signaling pathway, was performed in Fig.5B . It shows that the effects of HSDTT against KOA were connected with MAPK signaling pathway. The biological process that is linked to MAPK signaling pathway was displayed in Fig.6. We further investigated the influence of HSDTT on partly of this pathway in vivo.

\subsection{Investigation of the mechanism of HSDTT against KOA by experimental validation}

\subsubsection{The effect of HSDTT on cartilage tissue of knee in KOA rats}


As is shown in the Fig.7A-B,the surface of cartilage was smooth without cracks,the cartilage structure was clearly, such as regularly arranged chondrocyte and the complete tidemark in the HE staining of the control group. But in the staining of the model group, the surface of cartilage was damaged seriously, cells in transition zone arranged disordered with subchondral bone and erosion pannus formation. The makin score in model group is higher than the control group significantly. $(p<0.05$, Fig.7C). It confirms that the model of knee osteoarthritis was successfully established. After KOA rats treated with HSDTT for one

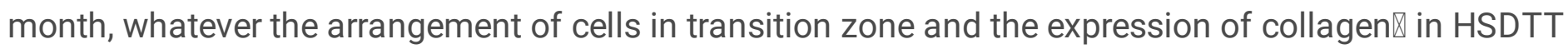
group were significantly improved when compared with the model group. $(p<0.05$,Fig.7D-G)

\subsubsection{The effects of HSDTT on the mRNA expression of p38 and p53}

Levels of p38 and p53 were further indicated that the changes of cartilage are related to the mechanism that HSDTT inhibits the $\mathrm{p} 53$ signaling pathway of KOA.In comparison with the control group,the mRNA expression of p38 and p53 were increased in model group $(p<0.01)$. However,after intervention with HSDTT, the protein expression of P38 and p53 decreased significantly $(\mathrm{p}<0.01, \mathrm{Fig} .7 \mathrm{H}-\mathrm{I})$.

\subsubsection{The effects of HSDTT against inflammation in KOA rats}

To verify the efficacy of HSDTT to KOA rats, knee swelling of the right legs were measured (Fig.8A). On day 7 , transverse diameters of the right knees in model group was significantly larger than that in control group ( $p<0.05)$. After the rats gavaged for 28 days, the knee joint diameter of HSDTT group was significantly smaller than that of model group on day $35(p<0.05)$. Furthermore, the synovium and synovial fluid were used to test the expression of inflammatory cytokine. As is shown in Fig.8B-C, the content of IL-1 $\beta$ and TNF- $\alpha$ in synovium significantly increased in model group when compared with control group $(p<0.01)$. Besides, in comparison with control group, rats in model group were observed with a significant increase in content of COX-2 and PGE-2 in synovial fluid( $p<0.01$,Fig.8D-E). By contrast, the expressions of IL-1 $\beta$,TNF-a,COX-2 and PGE-2 in HSDTT group are significantly lower than in model group $(p<0.01$,Fig.8B-E). It indicated that HSDTT has a good effect in alleviating inflammation of KOA.

\section{Discussion}

Nowadays, KOA has been a worldwide public healthy problem with its high disability rate among the people over 50 years old[26]. Therefore, a large number of researchers are devoted themselves to explore an effective method to halt the progression of KOA. As a common prescription of KOA, HSDTT has a good effect in early-stage of KOA, but the mechanisms of it are still unknown. Network pharmacology is a powerful tool which integrates other platforms and technologies to investigate the correlation between the ingredients of TCM and the targets of disease[27].In present study, the mechanism of HSDTT against KOA was analyzed by the network pharmacology and then validated by animal experiment.

In this study, 3096 potential targets of KOA and 1176 ingredients of HSDTT were analyzed through network pharmacology. The results indicated that HSDTT played a critical role in treating KOA by regulating some targets and signaling pathways. As the component of HSDTT, Atractylodes lancea 
(Thumb.)DC not only reduce the expression of inflammatory cytokines in serum, but also decreases the expression of beclin-1 protein in synovial tissue of rat[28].Moreover, Radix Angelicae pubescentis and licorice all have a great effect on alliviating inflammtory, and Radix Angelicae pubescentis has been shown to relieve pain by inhibiting the expression of TNF- $\mathrm{a}$ and IL-1 $\beta[29,30]$. Angelicae Sinensis Radix inhibits apoptosis of chondrocyte via suppressing JNK and p38MAPK pathways[31].

While some of components have been researched as a single herb in other studys, this decoction with all these components treating KOA remains to be explored. The results of network pharmacology indicated that the reason why can HSDTT treat KOA effectivly is that this decoction works through multiple components and targets. PPI network showed that the key targets of HSDTT in treatment of KOA were ATK1, GAPDH, IL-6, TP53, ALB, VEGFA, MAPK3, TNF, EGFR, MAPK1. We further revealed by G0 enrichment analysis and highlighted that HSDTT delayed the progression of KOA by regulating the activity of protein serine/threonine kinase, endopeptidase ,protein tyrosine kinase and so on. The KEGG enrichment analysis showed that PI3K-Akt signaling pathway, MAPK signaling pathway were the key pathways for HSDTT to treat KOA.

P38 MAPK is one of pathways in MAPK signaling pathway,which regulated the expression of proinflammatory cytokines such as IL-1 $\beta$ and TNF- $a$ to accelerate cartilage degeneration in KOA[32]. When growth factors and pro-inflammatory cytokines bind to corresponding receptors on the cell membrane, the pathway will be triggered. Then p38 will be phosphorylated and in turn active other inflammatory cytokines in downstream[9]. Recently, researchers have reported that synovial inflammantion is another important factor which can aggravate KOA by osteophytosis, cartilage degeneration and inflammation[33, 34]. some sudies have shown that overexpressions of COX-2 and PGE-2 in synovial tissues were probably induced by proinflammatory mediators like IL-1 $\beta$ and TNF- $a$ actived by p38 MAPK signaling pathway[35,36].

We further established a model of KOA in vivo by the injection in type II collagenase and comfirmed that HSDTT was effective in anti-inflammation. The results indicate that HSDTT is capable of delaying the progression of knee osteoarthritis, probably via suppressing the mRNA expressions of p38 and p53, lowering inflammatory cytokine (IL-1 $\beta$ and TNF- $\alpha$ ) and then decreasing the release of COX-2 and PGE-2, increasing collagen II.

This study had some limitations. Firstly,the network pharmacology is a new tool analyzed by the existing active ingredients of TCM, but the HSDTT decoction still has undiscovered ingredients. Secondly,we just selected only one signaling pathway identified through network pharmacological analysis to confirm the mechanism against KOA in vivo. Therefore, the machanisms that HSDTT treating for KOA analyzed by network pharmacology need more experiments in vivo and in vivo to comfirm.

\section{Conclusions}

In this study,a network pharmacological analysis was combined with experiment in vivo to clarify the mechanism of HSDTT against KOA. The results of network pharmacology show that HSDTT treats KOA 
by multiple targets and pathways. Further,via experimental evidence, we show that HSDTT delays the progression of KOA by regulating p38MAPK signaling pathway, thus alleviating inflammation and protecting cartilage.This present research provides evidence to support the clinical use of HSDTT for the treatment of KOA.

\section{Abbreviations}

HSDTT:Huashi Dingtong decoction; KOA:Knee osteoarthritis; TNF-a:tumor necrosis factor alpha; IL1ß:interleukin-1 beta; MAPK:Mitogenactivated protein kinase; ERK:extracellular regulated kinase; JNK:CJun N-terminal kinase; TCM:traditional Chinese medicine; GO:Gene ontology; KEGG:Kyoto Encyclopedia of Genes and Genomes; CSJ:Squama Manis (Chuan-Shan-Jia); RG:Cinnanmomi Cortex(Rou-Gui); SY:Rhizoma Dioscoreae (Shan-Yao); DG:Angelicae Sinensis Radix (Dang-Gui); WJP:Cortex Acanthopanacis (Wu-Jia-Pi); DH:Radix Angelicae Biseratae (Du-Huo); QH:Notopterygii Rhizoma Et Radix(Qiang-Huo); CZ:Atractylodes lancea(Thumb.)DC (Cang-Zhu); GC:licorice (Gan-Cao); H\&E:Hematoxylin-eosin staining; ELISA:Enzyme-linked immunosorbent assays; PCR:Polymerase Chain Reaction.

\section{Declarations}

\section{Acknowledgements}

Not applicable.

\section{Authors'contributions}

H-YG and L-JY serve as co-first authors. H-YG and L-JY designed this project; H-YG,YZ, Y-SZ,Z-LH and QDG conducted the experimental work;H-YG and L-JY and Y-YL collected the data. H-YG and L-JY analyzed the experimental data; $\mathrm{H}-\mathrm{YG}$ and L-JY contributed to drafting the manuscript. NL provided advice. All authors read and approved the final manuscript.

\section{Funding}

This work was funded by National Nature Science Foundation of China (No.81973880) and Natural Science Foundation of Fujian Province (No.2019J01349);

\section{Availability of data and materials}

The datasets used and/or analyzed during the current study are available from the corresponding author on reasonable request.

\section{Ethics approval and consent to participate}


All procedures of animal care and animal experiments were authorized by the Animal Ethical and Welfare Committee of Fujian University of Traditional Chinese Medicine (Protocol ID:NO.FUTCM-2019028) and conducted in compliance with Fujian University of Traditional Chinese Medicine

\section{Consent for publication}

Not applicable.

\section{Competing interests}

The authors declare that they have no competing interests.

\section{References}

1. Goldring M B , Goldring S R. Articular cartilage and subchondral bone in the pathogenesis of osteoarthritis. Annals of the New York Academy of ences, 2010, 1192: 230-237.

2. Siebuhr A , Bay-Jensen A , Jordan J , et al. Inflammation (or synovitis)-driven osteoarthritis: an opportunity for personalizing prognosis and treatment? Scandinavian Journal of Rheumatology, 2016:87-98.

3. Anna, Litwic, Mark H , et al. Epidemiology and burden of osteoarthritis. British medical bulletin, 2013: 185-199.

4. Kloppenburg M, Berenbaum F. Osteoarthritis year in review 2019: epidemiology and therapy. Osteoarthritis Cartilage, 2020,28(3):242-248.

5. Hawker G A, Croxford R, Bierman A S, et al. All-cause mortality and serious cardiovascular events in people with hip and knee osteoarthritis: a population based cohort study. PLoS One, 2014,9(3):e91286.

6. Felson D T . Clinical practice. Osteoarthritis of the knee. New England Journal of Medicine, 2006, 354(8):841-848.

7. Loeser R F, Goldring S R, Scanzello C R, et al. Osteoarthritis: a disease of the joint as an organ. Arthritis Rheum, 2012,64(6):1697-1707.

8. Berenbaum $\mathrm{F}$. Osteoarthritis as an inflammatory disease (osteoarthritis is not osteoarthrosis!). Osteoarthritis \& Cartilage, 2013, 21( 1):16-21.

9. Chow YY, Chin KY. The Role of Inflammation in the Pathogenesis of Osteoarthritis. Mediators Inflamm. 2020;doi:10.1155/2020/8293921

10. Loeser R F , Erickson E A , Long D L . Mitogen-activated protein kinases as therapeutic targets in osteoarthritis. Current Opinion in Rheumatology, 2008, 20(5):581-586.

11. Zhang $Y$, Pizzute $T$, Pei M . A review of crosstalk between MAPK and Wnt signals and its impact on cartilage regeneration. Cell \& Tissue Research, 2014, 358(3):633-649.

12. Radons, Jürgen, Bosserhoff A, Grässel, Susanne, et al. p38MAPK mediates IL-1-induced downregulation of aggrecan gene expression in human chondrocytes. International Journal of Molecular 
Medicine, 2006,17(4):661-668.

13. Ma N, Teng X, Zheng $Q$, et al. The regulatory mechanism of p38/MAPK in the chondrogenic differentiation from bone marrow mesenchymal stem cells. Journal of Orthopaedic Surgery and Research, 2019, 14(1):434.

14. Shanghai Institute of Organic Chemistry of CAS. Chemistry Database[DB/OL]. 19782020.http://www.organchem.csdb.cn.

15. Ru J , Li P , Wang J , et al. TCMSP: a database of systems pharmacology for drug discovery from herbal medicines. J Cheminform, 2014, 6(1):13.

16. Kim S, Chen J, Cheng T, et al. PubChem 2019 update: improved access to chemical data. Nucleic Acids Res, 2019,47(D1):D1102-D1109.

17. Daina A, Michielin O ,Zoete V . SwissADME: a free web tool to evaluate pharmacokinetics, druglikeness and medicinal chemistry friendliness of small molecules. entific Reports, 2017, 7:42717.

18. Jianliang Li,Chaolu Wang,Shuzheng Cai,et al. The effect mechanism of whole pomegranate treated for functional gastrointestinal diseases by 'zhu-wei-huo' based on network pharmacology. Chinese Traditional Patent Medicine. 41(09) (2019) 2240-2245.[In Chinese]

19. Wang Y, Zhang S, Li F, et al. Therapeutic target database 2020: enriched resource for facilitating research and early development of targeted therapeutics. Nucleic Acids Res, 2020,48(D1):D1031D1041.

20. Hamosh A, Scott A F, Amberger J S, et al.Online Mendelian Inheritance in Man (OMIM) , a knowledgebase of human genes and genetic disorders[J].Nucleic Acids Rer, 2005, 33 (1) :514.

21. Stelzer G, Rosen N, Plaschkes I, et al. The GeneCards Suite: From Gene Data Mining to Disease Genome Sequence Analyses. Current Protocols in Bioinformatics, 2016, 54.

22. Antoine D , Olivier M , Vincent Z . SwissTargetPrediction: updated data and new features for efficient prediction of protein targets of small molecules. Nucleic Acids Research, 2019(W1):W1.

23. Shannon P, Markiel A, Ozier O, et al. Cytoscape: A Software Environment for Integrated Models of Biomolecular Interaction Networks. Genome Res, 2003,13(11):2498-2504.

24. Damian S, Morris J H , Helen C, et al. The STRING database in 2017: quality-controlled proteinprotein association networks, made broadly accessible. Nuclc Acids Research:D362-D368.

25. Yu G , Wang L G , Han Y, et al. clusterProfiler: an R package for comparing biological themes among gene clusters. Omics-a Journal of Integrative Biology, 2012, 16(5):284-287.

26. Liu Q, Wang S, Lin J, et al. The burden for knee osteoarthritis among Chinese elderly: estimates from a nationally representative study. Osteoarthritis Cartilage, 2018,26(12):1636-1642.

27. Cai F F , Bian Y Q , Wu R, et al. Yinchenhao decoction suppresses rat liver fibrosis involved in an apoptosis regulation mechanism based on network pharmacology and transcriptomic analysis. Biomedicine \& Pharmacotherapy, 2019, 114.

28. Liu R, Tao E, Yu S, et al. The Suppressive Effects of the Petroleum Ether Fraction from Atractylodes lancea (Thunb.) DC. On a Collagen-Induced Arthritis Model. Phytother Res, 2016,30(10):1672-1679. 
29. Li R, Zhao C, Yao M, et al. Analgesic effect of coumarins from Radix angelicae pubescentis is mediated by inflammatory factors and TRPV1 in a spared nerve injury model of neuropathic pain. $J$ Ethnopharmacol, 2017,195:81-88.

30. Jia T, Qiao J, Guan D, et al. Anti-Inflammatory Effects of Licochalcone A on IL-1 $\beta$-Stimulated Human Osteoarthritis Inflammation, 2017,40(6):1894-1902.

31. Zhou Y, Ming J, Li Y, et al. Ligustilide attenuates nitric oxide-induced apoptosis in rat chondrocytes and cartilage degradation via inhibiting JNK and p38 MAPK pathways. J Cell Mol Med, 2019,23(5):3357-3368.

32. Liao $C R$, Wang $S N$, Zhu $S Y$, et al. Advanced oxidation protein products increase TNF-a and IL-1 $\beta$ expression in chondrocytes via NADPH oxidase 4 and accelerate cartilage degeneration in osteoarthritis progression. Redox Biol, 2020,28:101306.

33. Scanzello C R, Goldring S R. The role of synovitis in osteoarthritis pathogenesis. Bone, 2012, 51(2):249-257.

34. Faour $\mathrm{W} \mathrm{H}, \mathrm{He} \mathrm{Y,} \mathrm{He} \mathrm{Q} \mathrm{W,} \mathrm{et} \mathrm{al.} \mathrm{Prostaglandin} \mathrm{E(2)} \mathrm{regulates} \mathrm{the} \mathrm{level} \mathrm{and} \mathrm{stability} \mathrm{of} \mathrm{cyclooxygenase-}$ 2 mRNA through activation of p38 mitogen-activated protein kinase in interleukin-1 beta-treated human synovial fibroblasts. J Biol Chem, 2001,276(34):31720-31731.

35. Martel-Pelletier J, Pelletier J P, Fahmi H. Cyclooxygenase-2 and prostaglandins in articular tissues. Semin Arthritis Rheum, 2003,33(3):155-167.

\section{Figures}

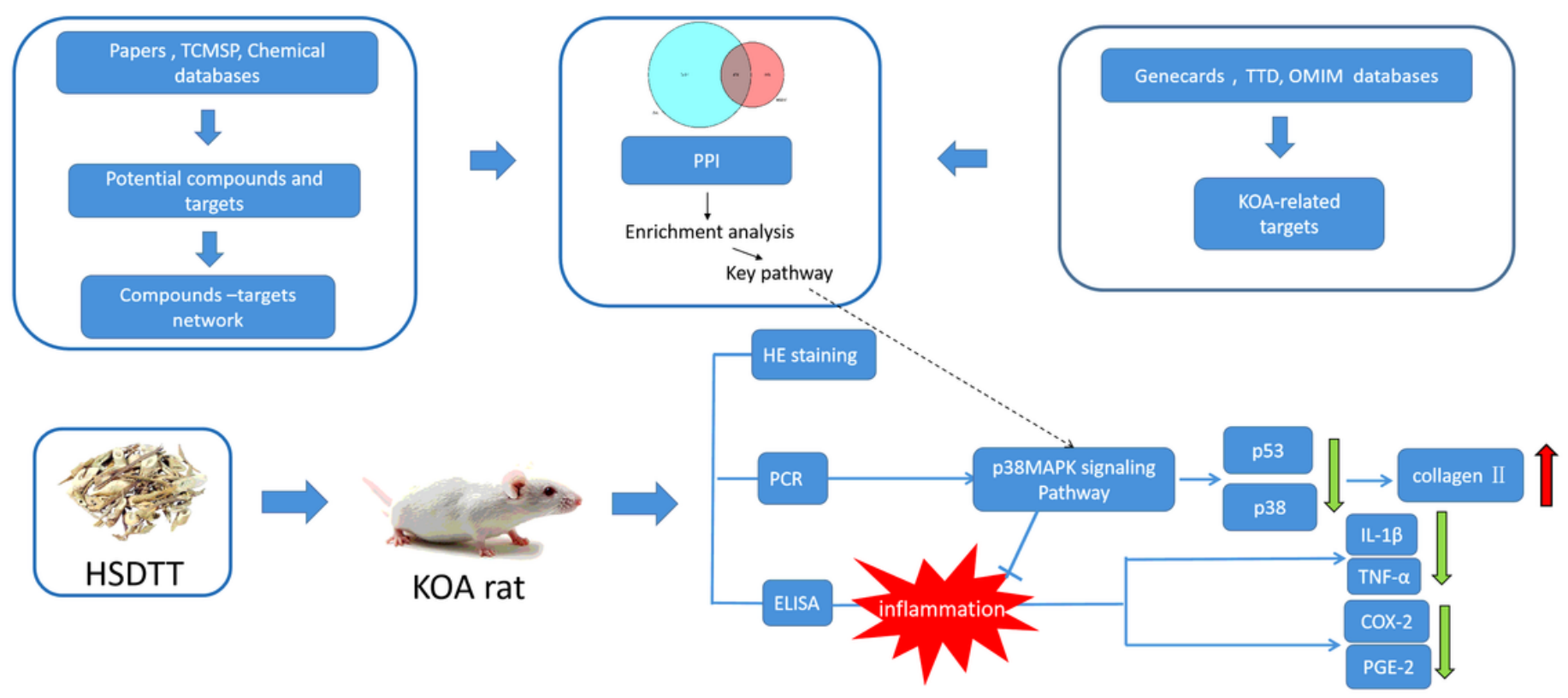

\section{Figure 1}

Flowchart of designed analysis of HSDTT against inflammation. 


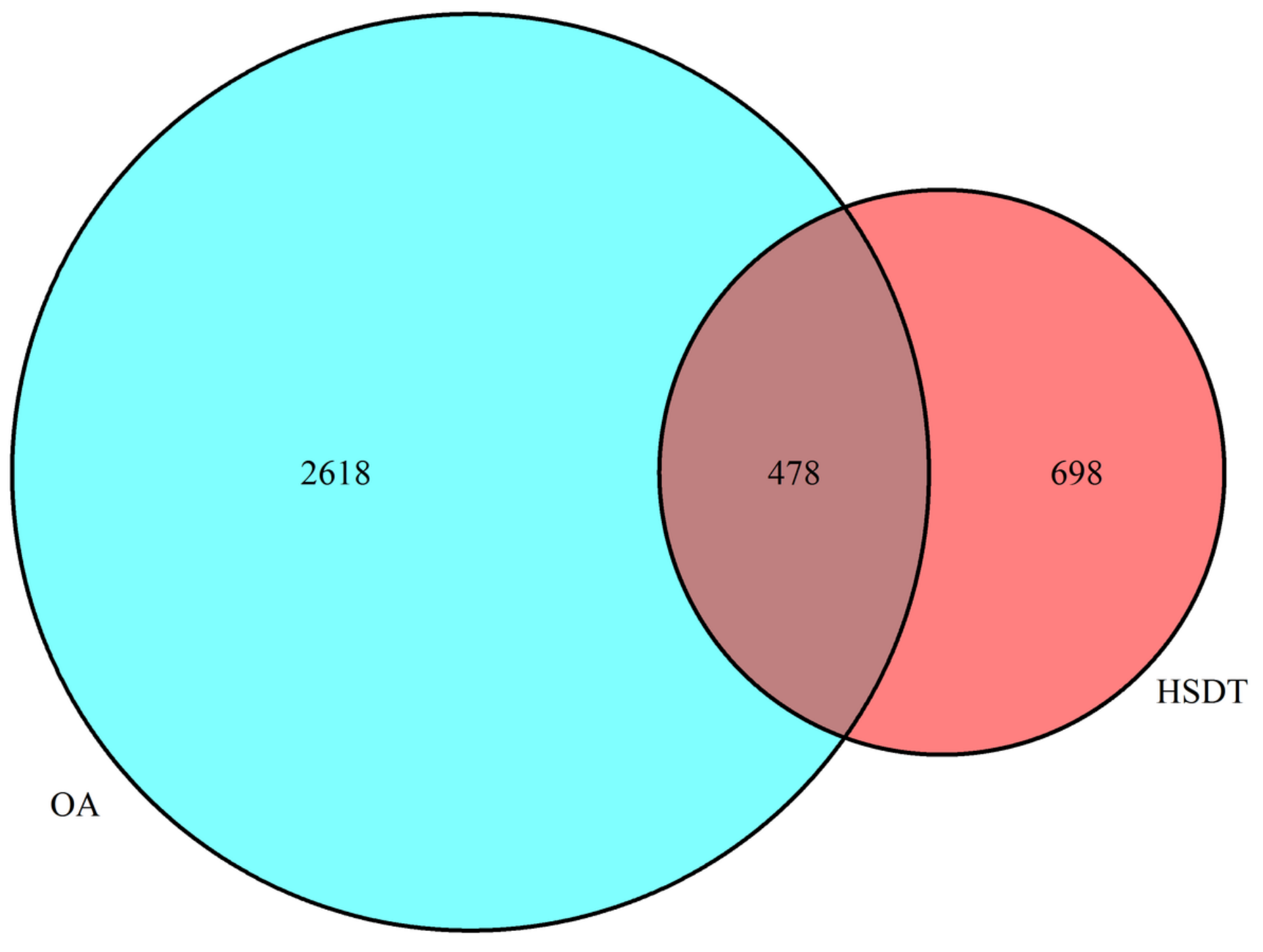

Figure 2

Venn diagram graph .Venn diagram of candidate targets in KOA and HSDTT. 


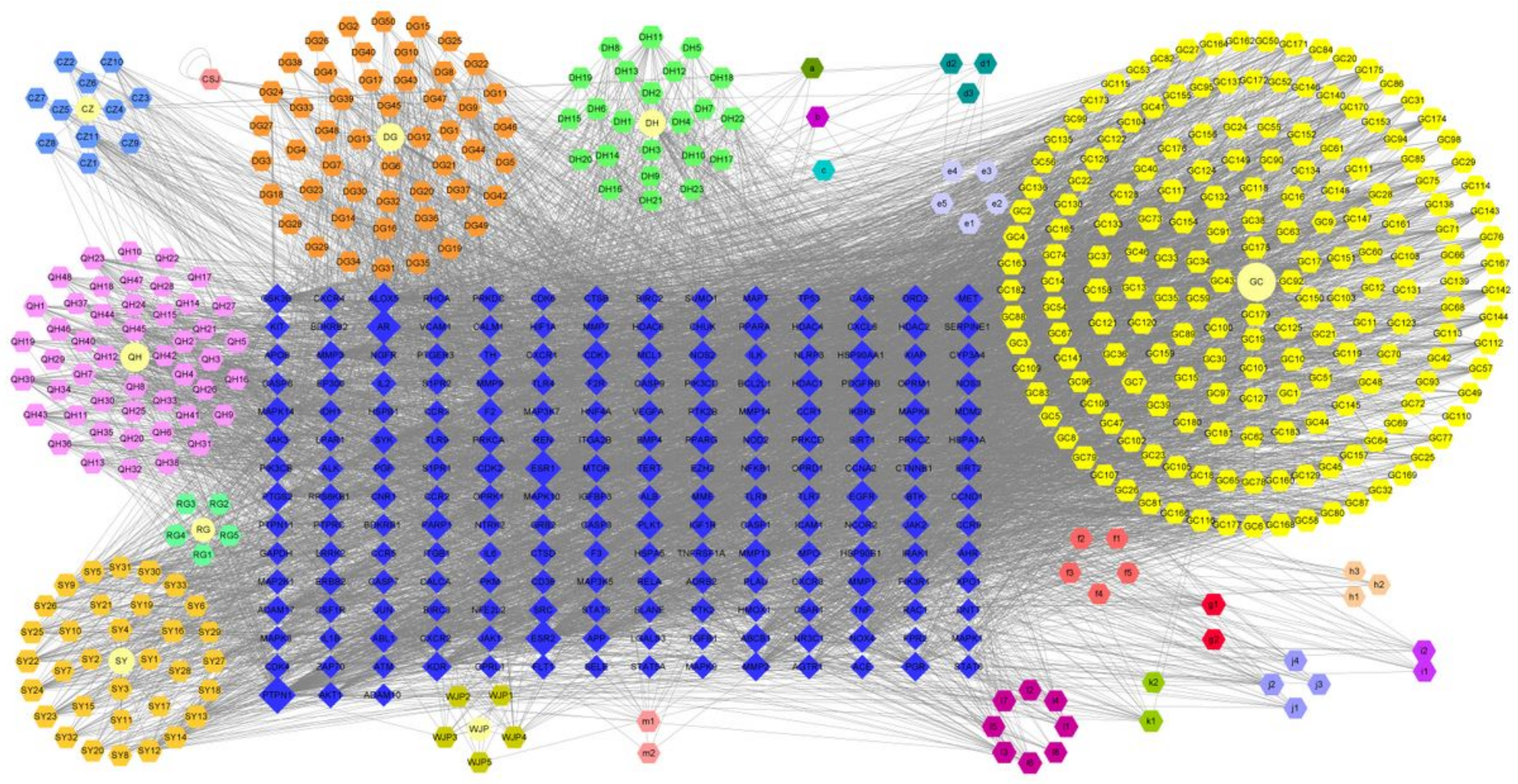

\section{Figure 3}

Drug-Ingredient-target network. The Rhombus represents the first 200 targets of HSDTT for treatment of KOA.Hexagons with different colors represents different ingredients (the name of each ingredients can search in Table.S1.) of HSDTT. The circle represents different drugs of HSDTT.

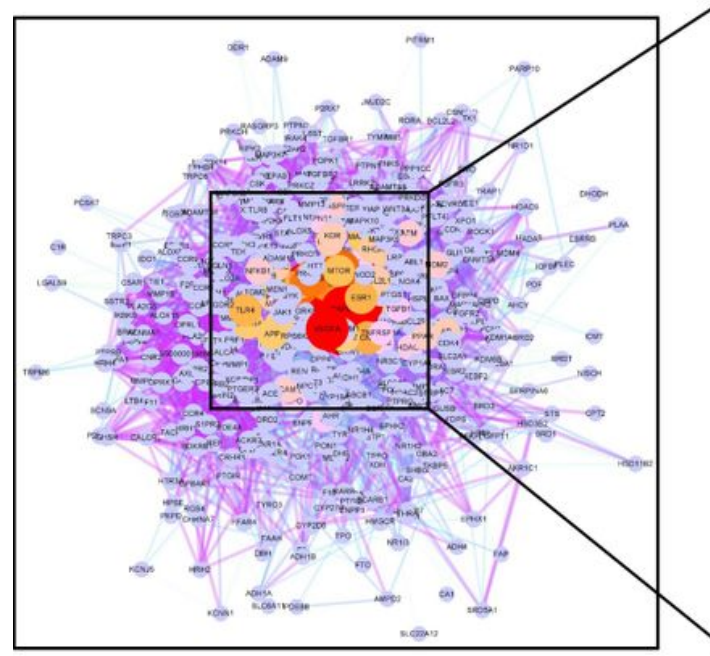

(A) 478 Nodes 11506 edges

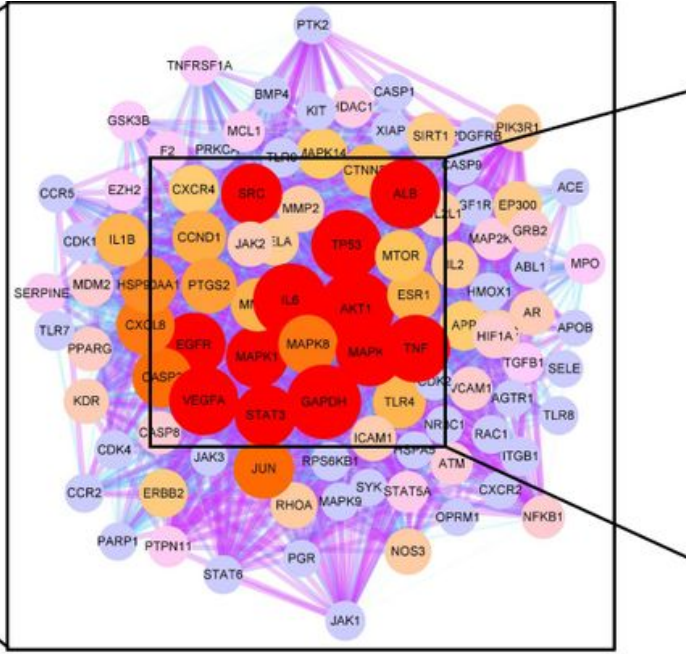

(B) 100 Nodes 2980edges

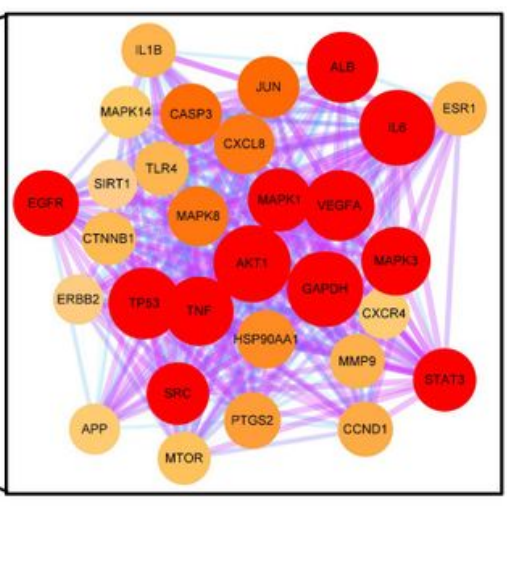

(C) 30Nodes 420edges

\section{Figure 4}

The protein-protein interaction (PPI) network.(A) The PPI network containing 478 nodes and 11506 edges. (B)The PPI network for the candidate targets ranked in the top 100. The PPI network containing 100 
nodes and 2980 edges.(C)The PPI network for the candidate targets ranked in the top 30.The PPI network containing 30nodes and 420 edges.

A

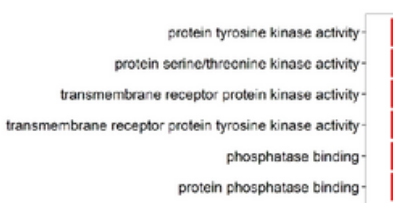

nucleat receptor activity.

transcription factor activity, direct ligand regulated sequence-specific DNA tinding-

endopeptidase activity-

steroid hormone receptor activity-

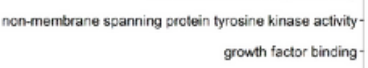

peptide receptor activity

G.protein coupled peptide receptor activity.

metalloendopeptidase activity.

chemokine tinding.

c.c chemokine recoptor activity

heat shock protein binding.

c.C chemokine binding-
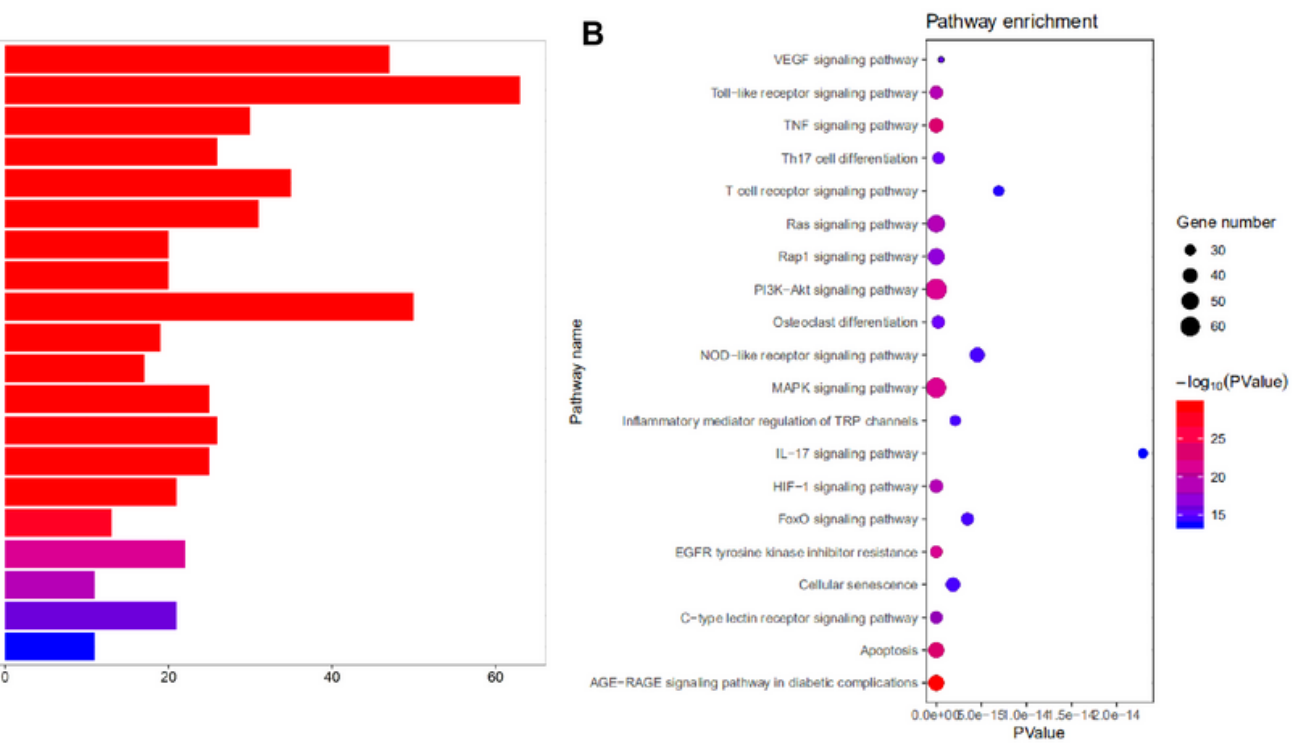

\section{Figure 5}

Functional analysis. A. The GO enrichment analysis of the 478 targets with bar plot. B. The KEGG pathway enrichment analysis of 478 targets with dot plot. 


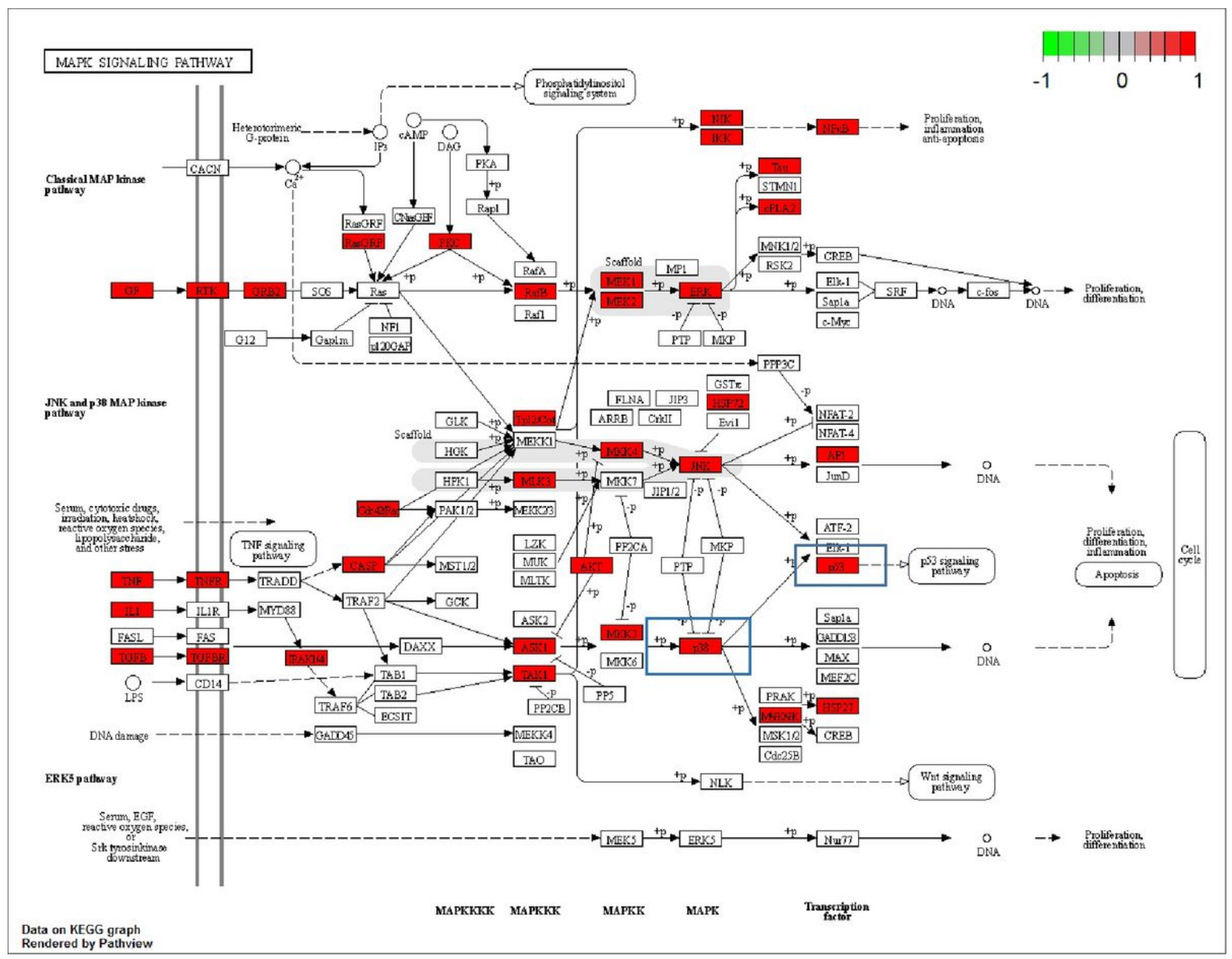

\section{Figure 6}

The pharmacological mechanism of HSDTT against KOA. MAPK signaling pathway play an important role in treating KOA with HSDTT. 


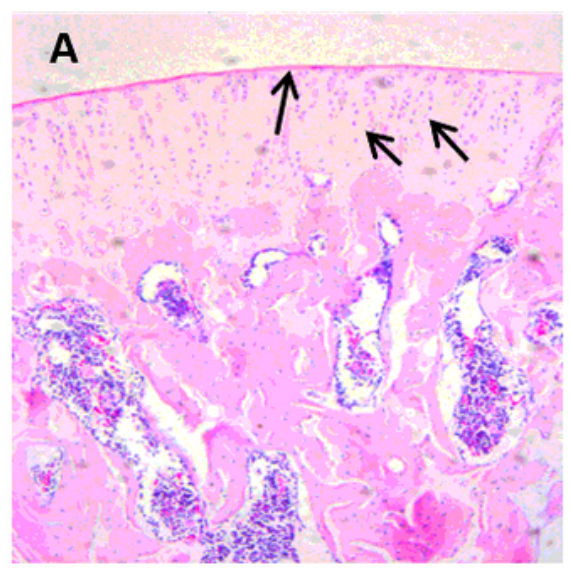

\section{Control}

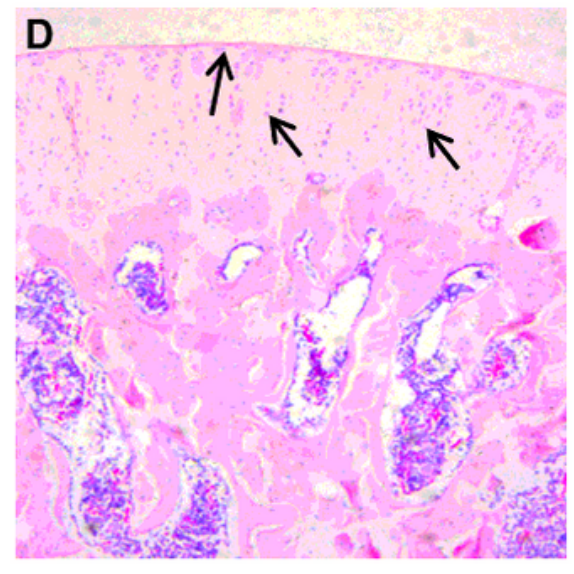

Control

G

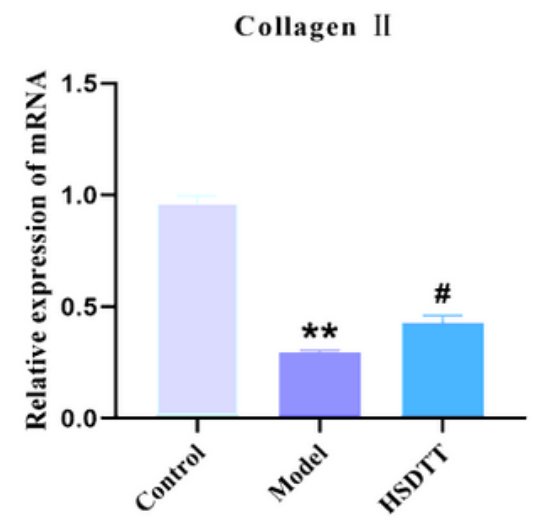

B

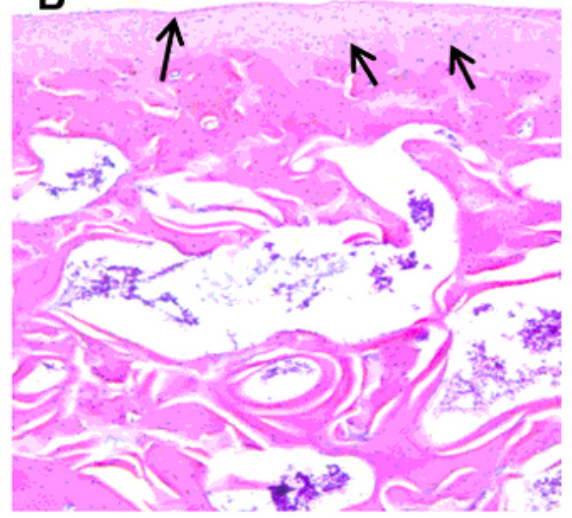

Model

E

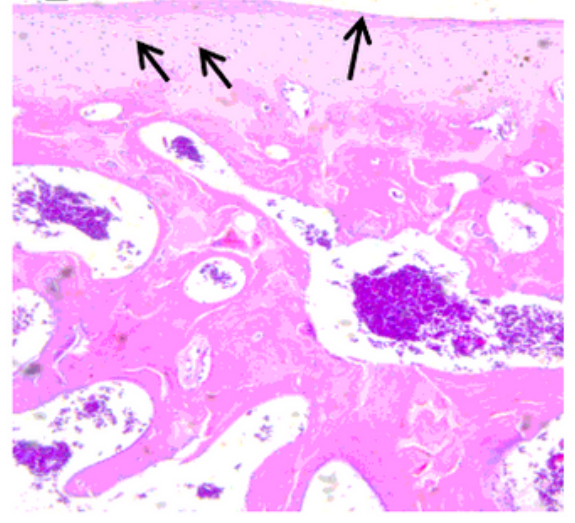

Model

H

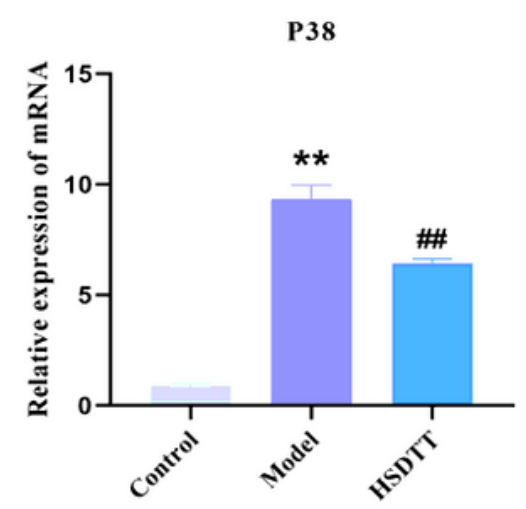

C

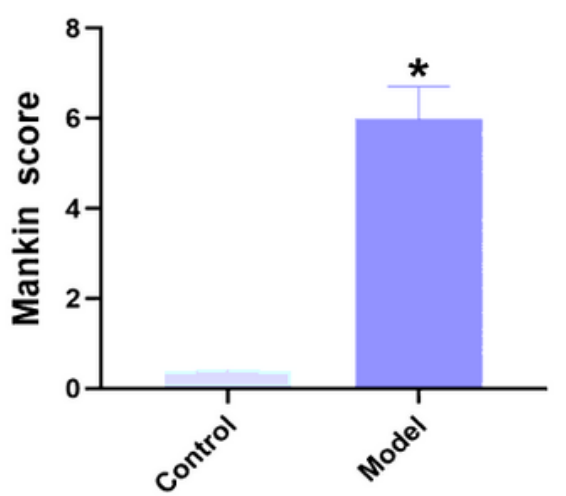

F

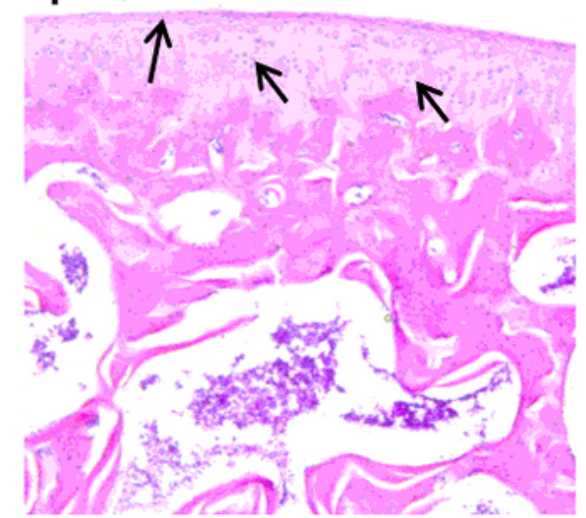

KOA

I

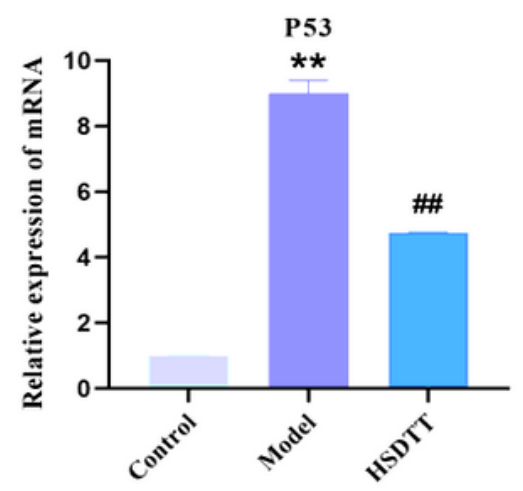

\section{Figure 7}

The effect of administration of HSDTT on cartilage in knee osteoarthritis rats. (A-B) Histopathological examination of knee joints after KOA model successfully established (100x). (D) Mankin score were significantly increased after model successfully established. ${ }^{*} p<0.01$ vs. control group;*p $<0.05$ vs. the control group; (D-F) Histopathological examination of knee joints of knee joints after KOA rats gavaged 
for 28 days; (G-I)The relative expression of mRNA of collagen $\rrbracket, p 38$, p53 in cartilage.Values shown are mean $\pm S D(n=8) ; * \star p<0.01$ vs. control group; $\# \# p<0.01$ vs. the model group.

A

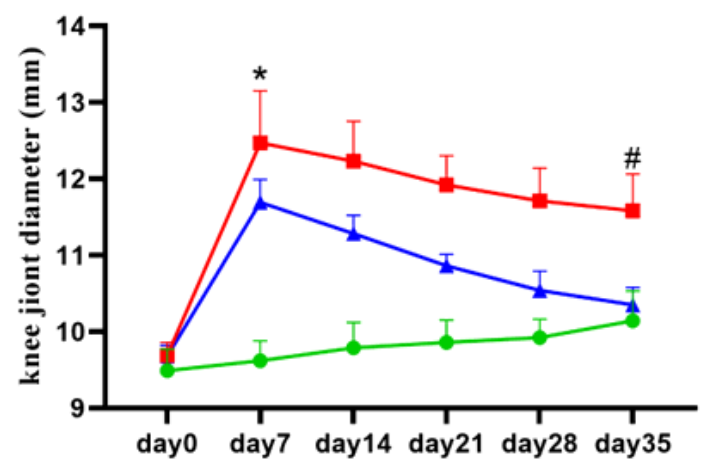

C

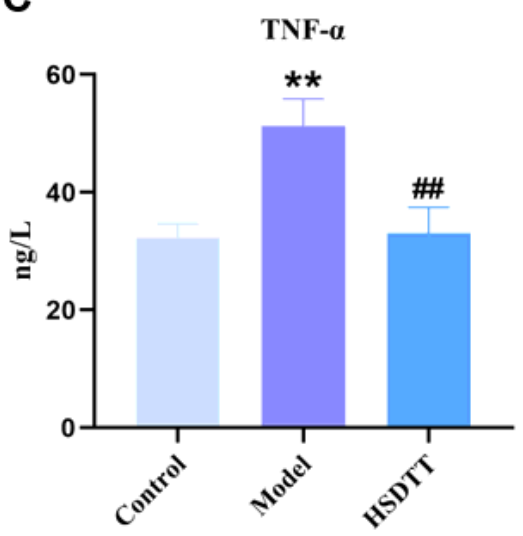

$\rightarrow$ Control

$\rightarrow$ Model

$\neq$ HSDTT

D

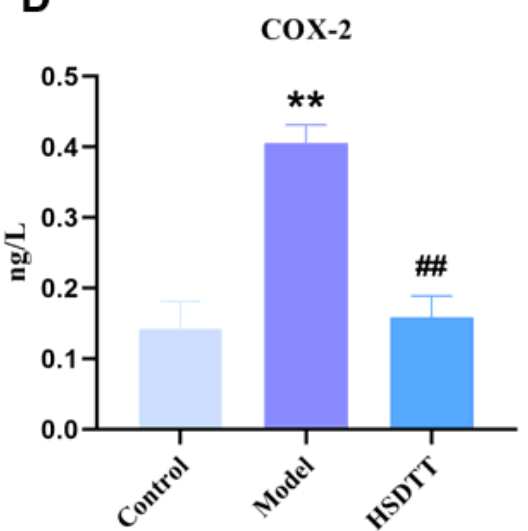

B

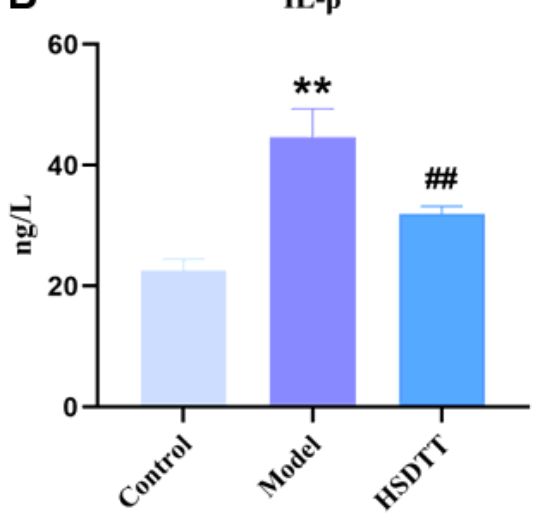

E

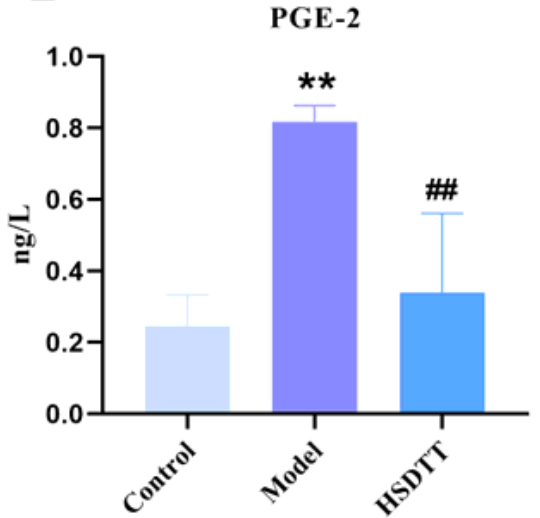

\section{Figure 8}

The effect of administration of HSDTT against inflammation in KOA rats. (A)The severity of inflammation evaluated by the transverse diameters of the right knees. ${ }^{*} p<0.05 v s$. the control group; \#p $<0.05$ vs. the model group.(B-C) The content of IL-1 $\beta$,TNF- $a$ in synovium.(D-E) The content of COX-2,PGE2 in synovial fluid.Values shown are mean $\pm S D(n=8) ; * \star p<0.01$ vs. control group; $\# \# p<0.01$ vs. the model group.

\section{Supplementary Files}

This is a list of supplementary files associated with this preprint. Click to download.

- Theinformationof478effectivetargetsrelatedKOA.docx

- AlistofactiveingredientsinHSDTT.docx 